\title{
Sustained-release Mitomycin C Hydrogel Formulation
}

National Cancer Institute

\section{Source}

National Cancer Institute. Sustained-release Mitomycin C Hydrogel Formulation. NCI

Thesaurus. Code C126642.

A sustained-release (SR) hydrogel polymer-based formulation containing the antineoplastic antibiotic mitomycin C (MMC), with potential antineoplastic activity. Upon local administration of the SR MMC hydrog el formulation to the upper urinary tract via a ureteral catheter, the gel solidifies and deposits MMC locally to prevent the excretion of this chemotherapeutic agent via urinary flow. In turn, MMC alkylates DNA, and produces interstrand DNA cross-links, thereby inhibiting DNA synthesis. Due to its reverse thermalgelation properties, this gel is able to stay in a liquid state at cold temperatures and solidifies at body temperature. This allows for increased accumulation of MMC locally in the upper urinary tract which leads to increased efficacy compared to standard intravesical delivery of MMC for upper tract urothelial carcinoma (UT UC). 